\title{
La evaluación de la sostenibilidad de los destinos turísticos en su relación con el enfoque a procesos.
}

The evaluation of the sustainability of tourist destinations in relation to the process approach.

Martha Omara Robert Beatón. ${ }^{1}$, Maite Echarri Chávez. ${ }^{2}$ \& José Antonio Acevedo Suárez $^{3}$

\begin{abstract}
The fast development of the tourist destinies and the complexity of the processes than in he attend, demand the search of tools, methods and models for the evaluation of his sustainability. In this sense the present work aims at weighing the validity of the foundations from the focus to processes to evaluate the sustainability of the tourist destinies, taking like base the experiences in the business sector that refers the scientific looked up literature. Using the methods of synthesis of the topical bibliography fundamentally object of study, and the comparative analysis. The results of this analysis demonstrated that tools models and methods that are used at the present time do not allow evaluating the sustainability of the destinies from an interdimensional focus, intradimensional and multidimensional that he integrates the complex dynamics that take place in the same, laying the foundations to accomplish investigations from other areas of the knowledge in the search of instruments pointed to acknowledge to and to understand the holistic vision of the sustainability in the tourist destinies
\end{abstract}

Keywords: Sustainability, Focus To Processes , Tourist Destinations

\section{Resumen}

\footnotetext{
1 Vicedecana de la Facultad de Turismo, Universidad de La Habana. Cuba. Ingeniera Agrónoma, Universidad de Granma, Cuba, Máster en Gestión Turística, y Doctora en Ciencias Económicas, Universidad de La Habana, Cuba.omara_robert@ ftur.uh.cu

${ }^{2}$ Coordinadora de la Maestría en Gestión Turística, Facultad de Turismo, Universidad de La Habana, Cuba. Licenciada en Geografía, Máster en Geografía, Medio ambiente y ordenamiento territorial y Doctora en Ciencias Geográficas, Universidad de La Habana, Cuba. maite_echarri@ftur.uh.cu

${ }^{3}$ Vicerrector de la Universidad tecnológicas de La Habana, Cuba. Ingeniero Industrial, Universidad de La Habana, Máster en Gestión empresarial, universidad técnica ktnizer, Alemania y Doctor en Ciencias Universidad Tecnológicas de La Habana, Cuba acebedo@tesla.cujae.edu.cu
} 
El rápido desarrollo de los destinos turísticos y la complejidad de los procesos que en el concurren, exige la búsqueda de herramientas, métodos y modelos para la evaluación de su sostenibilidad. En este sentido el presente trabajo tiene como objetivo valorar la validez de los basamentos del enfoque a procesos para evaluar la sostenibilidad de los destinos turísticos, tomando como base las experiencias en el sector empresarial que refiere la literatura científica consultada. Utilizando fundamentalmente los métodos de síntesis de la bibliografía sobre el tema objeto de estudio, y el análisis comparativo. Los resultados de este análisis demostraron que las herramientas modelos y métodos que se utilizan en la actualidad no permiten evaluar la sostenibilidad de los destinos desde un enfoque interdimensional, intradimensional y multidimensional que integre las dinámicas complejas que se producen en los mismos, sentando las bases para realizar investigaciones desde otras áreas del conocimiento en la búsqueda de instrumentos dirigidos a reconocer y a comprender la visión holística de la sostenibilidad en los destinos turísticos.

Palabras Clave: Sostenibilidad, Evaluacion, Enfoque a Procesos, Destinos Turísticos.

\section{Introducción}

Con el informe Brundtland se incluyó en la agenda internacional la concepción del desarrollo sostenible, entendido como "el desarrollo que satisface las necesidades actuales sin comprometer las capacidades de las generaciones futuras de satisfacer las suyas" (WCED1987) este concepto fue retomado en la Cumbre de la Tierra, celebrada en Río de Janeiro en 1992, momento a partir del cual se le comienza a otorgar mayor importancia a sus principios básicos. En esta se modifica la definición original, que estaba centrada en la preservación del medio ambiente y el consumo prudente de los recursos naturales no renovables, hacia la idea de "tres pilares" que deben conciliarse en una perspectiva única: el progreso económico, la justicia social y la preservación del medio ambiente.

A partir de la Conferencia de Río de 1992, se debate de qué forma el turismo debe desempeñar un papel dentro del desarrollo sostenible. A esta le han sucedido otros eventos que han determinado el camino a seguir en la búsqueda de la sostenibilidad turística; es así como en 1995 se elabora la Carta de Lanzarote, en el marco de la Conferencia Mundial de Turismo Sostenible, en 1996, la Agenda 21 para la Industria de Viajes y Turismo; en el año 2000 se establecen los Objetivos de Desarrollo del Milenio, (ODM), son ocho con el propósito del desarrollo humano, en el 2002 la Cumbre Mundial se pronuncia por un programa de desarrollo sostenible y en el 2012, en la Conferencia sobre Desarrollo Sostenible Río+20 se creó un grupo de trabajo para desarrollar los Objetivos de Desarrollo Sostenible, estableciendo 17 planteando el horizonte 2015-2030 (Anton et al., 2012; Blay, 2012; Pérez de las Heras 2004; Chiappe y Pineiro, 2003).

Por su parte la comunidad científica a nivel internacional promueve investigaciones encaminadas al desarrollo sostenible y a la sostenibilidad turística, significando los mayores avances hacia la definición de instrumentos y herramientas para su evaluación, 
sin embargo los mismos aun evidencian carencias o vacíos para evaluar las dinámicas complejas que concurren en las interrelaciones de un destino. Estas interrelaciones se manifiestan en todas las fases del proceso de gestión del mismo, lo que deviene en la complejidad del diagnóstico o evaluación de los avances hacia la sostenibilidad o no, en aras de tomar decisiones pertinentes en función de los objetivos de desarrollo determinados (Torres, 2016; Um, 2010 Ramos y Caeiro, 2010; Pulido y Sánchez, 2009; Pérez y Nel-lo, 2009; Pérez et. al, 2009; Blancas et al., 2009 Martínez, López, e Santos, 2007; Choi y Sirikaya, 2005).

En Cuba la mayoría de los estudios sobre sostenibilidad turística de destinos han sido realizados a partir de la determinación y evaluación de indicadores divididos de acuerdo a las dimensiones de la sostenibilidad. Esto ha estado determinado por la elaboración de la propuesta de indicadores de sostenibilidad para el turismo en Cuba, documento elaborado de conjunto entre el Ministerio de Turismo (MINTUR) y el Ministerio de Ciencia Tecnología y Medio Ambiente (CITMA) cubanos en el año 2003, el cual, aunque todavía insuficiente, ha constituido pauta para el desarrollo de las investigaciones más recientes con respecto a este tema en el país.

Entre esas investigaciones se encuentran las realizadas por Blanco (2016) para el destino Cuba, Delis y Echarri (2015) en el Centro Histórico de La Habana, sobre varios destinos de naturaleza cubanos. Estos estudios, aunque han coincidido en el método o herramienta a utilizar, difieren, al igual que los estudios internacionales sobre este tema, en cuanto al enfoque aplicado, así como en el proceso de determinación de los indicadores y, por supuesto, en los indicadores determinados para cada caso.

Otros estudios en Cuba sobre esta misma dinámica de análisis han versado sobre la conceptualización del sistema turístico (Martín 2006), su desarrollo (Figueras, 2008) y aspectos concretos alrededor de sus componentes en función de proponer metodologías para su pronóstico (Delgado, 2014; La Serna, 2014; Perelló, 2005), su desempeño económico y espacial y los impactos a nivel social (Vargas, 2013; Pérez, 2013; Pérez, 2011; Echarri, 2006). Estos análisis asumen al destino como una gran organización que basa su gestión en las funciones que desempeñan los diferentes actores que estén presentes en el mismo. La gestión por funciones definen y establecen áreas de trabajo mediante la creación de varias unidades especializadas y jerarquizadas que son responsables de diferentes actividades específicas (Villazón, 2015; Williams, 2008; Taylor y Ford 1980) pero que no permiten entender al destino de manera integrada, generando problemas de fragmentación que impiden visualizar y gestionar las interacciones que deben existir entre las partes.

El enfoque funcional, si bien provee claridad en los objetivos particulares y su procedimiento, encuentra dificultades para entender las interrelaciones entre los componentes, orientándose hacia el fin específico que le ha sido asignado a cada unidad.

Esta debilidad puede ser corregida asumiendo un enfoque de gestión por procesos, el cual provee a las organizaciones de las secuencias de actividades que deben realizar en 
conjunto conscientes de sus interdependencias con el propósito de que puedan cumplir una misión compartida.

Si bien el enfoque por procesos ha sido aplicado a la gestión de las organizaciones empresariales concebidas como un sistema, no se han aprovechado la validez de sus principios en el análisis de la sostenibilidad de los destinos ya que los enfoques prevalecientes se basan en modelos tradicionalmente funcionales como es el caso del modelo presión estado respuesta (PER. El modelo PER está basado en una lógica de causalidad: las actividades humanas ejercen presiones sobre el ambiente y cambian la calidad y cantidad de los recursos naturales (estado). Asimismo, la sociedad responde a estos cambios a través de políticas ambientales, económicas y sectoriales (respuestas) (OCDE, 1993). Si bien resulta un esquema lógico en términos de la relación entre presiones, estado y acciones, sugiere una relación lineal de la interacción entre las actividades humanas y el ambiente, ocultando los aspectos complejos de estas interacciones, presentándose como una oportunidad para la inclusión del enfoque por procesos, de modo tal que estas relaciones causales sean analizadas a partir de sus funciones.

Ante la multiplicidad de enfoques, modelos y herramientas, así como las diferencias entre las investigaciones realizadas siguiendo los mismos métodos para evaluar la sostenibilidad del desarrollo de los destinos turísticos, se hace necesario establecer una base uniforme para definir niveles de sostenibilidad y al mismo tiempo permita establecer comparaciones espaciales y temporales entre diferentes destinos turísticos, por lo que se plantea como objetivo general de esta investigación: valorar los principios del enfoque a procesos en la evaluación de la gestión sostenible de los destinos turísticos.

\section{Metodología}

La investigación científica se concibe como un conjunto de procesos sistemáticos y empíricos que se aplican al estudio de un fenómeno; es dinámica, cambiante y evolutiva (Hernández, Fernández \& Baptista, 2006). Específicamente, la investigación turística está concebida como la formulación de preguntas, la sistemática colección de información para responder a esas preguntas y la organización y análisis de los datos con el fin de obtener pautas de comportamiento, relaciones y tendencias que ayuden al entendimiento del sistema, a la toma de decisiones o a la construcción de predicciones bajo el abanico de varios escenarios alternativos de futuro (Sancho, 2008). Teniendo en cuenta su alcance, y siguiendo a Perelló (2011), la investigación es de tipo descriptiva, pues como su tipología lo indica en el desarrollo del estudio científico se describe lo más preciso posible al fenómeno que se investiga; al mismo tiempo Hernández, Fernández y Baptista (2006), coinciden en que los estudios descriptivos, evalúan o recolectan datos sobre diversos conceptos [variables], aspectos, dimensiones o componentes del fenómeno a investigar. Es por ello que en este tipo de estudios el investigador debe ser capaz de definir, o al menos visualizar, conceptos, principios y tendencias.

La trayectoria metodológica de la investigación Se centró en un enfoque cualicuantitativo, de tipo descriptiva, analítica-exploratoria. Comprendió 2 fases partiendo en 
la fase 1 , de los antecedentes, fundamentando desde la línea de investigación desarrollo sostenibles de destinos turísticos de la Facultad de Turismo que asume un proyecto encaminado hacia la evaluación sostenible con un enfoque a procesos, donde se han generado investigaciones que abarcan una amplia gama de estudios que constituyen problemas reales en la operación de entidades y destinos turísticos, conduciendo a resultados que evidencian la necesidad de nuevos instrumentos desde otras áreas del conocimiento dirigidos a reconocer y a comprender la visión holística de la sostenibilidad en los destinos turísticos.

En esta fase predominó como principal método el análisis bibliográfico que consistió en la recopilación de información procedente de la literatura consultada, reveló conceptos interpretados y definidos desde diversas perspectivas, que acompañado de otros métodos como el inductivo -deductivo, sistémico, dialectico, histórico- lógico, permitieron estructurar estos aspectos en el cuerpo de la investigación; presentados además desde lo simple a lo complejo y siguiendo los procesos de pensamientos lógicos, lineales, secuenciales y relacionables.

Esto condujo a dos aseveraciones en relación con los destinos turísticos, primero, que los principios que rigen las organizaciones empresariales son coherentes con los destinos, entendidos como una organización sistémica, y segundo, que desde las propias característica que ellos revelan como una organización sistémica compartiendo las mismas premisas, se sustenta la justificación de adaptar el basamento del enfoque a procesos a la evaluación de su sostenibilidad.

En la fase 2 se definen los elementos metodológicos para adaptar los basamentos del enfoque a procesos en la evaluación de la sostenibilidad de los destinos turísticos. A partir de sistematizar y realizar un análisis comparativo de las experiencias en la aplicación de metodologías para este enfoque en organizaciones empresariales, que de conjunto con la aplicación de otros métodos como el Delphi, la observación, la entrevista, el análisis estadístico descriptivo y talleres facilitaron determinar los procesos, subprocesos y los grupos de interés que concurren en un destino turístico, utilizando los métodos de encuestas, observación, talleres,. Para adaptar los basamentos del enfoque a procesos en la evaluación de la sostenibilidad de los destinos turísticos.

\section{Resultados}

\section{El destino turístico como una organización sistémica.}

La norma internacional ISO 9001:2015 define a la organización como una persona o grupo de personas que tienen sus propias funciones y responsabilidades, autoridades y relaciones para el logro de sus objetivos.

Las organizaciones son entidades creadas por la sociedad, o por grupos de personas, con el fin de lograr en forma conjunta un resultado, un producto, un bien o un servicio. El reto consiste en que ésta opere adecuadamente y logre con éxito el propósito o misión para la cual fue creada (Coaguila, 2017; Rojas, 2014; Fraguela et al., 2012; Thompson, 2012).Señalando además que su creación parte de dos premisas: 
1) Que sean constituidas por el Estado o un grupo de personas que tienen una misión compartida o propósito común que cumplir;

2) Que para cumplir con esa misión lo hagan a través de procesos.

De acuerdo a la teoría de sistemas, la organización es un conjunto formado por partes interrelacionadas que constituyen un todo coherente y desarrolla un marco sistemático para la descripción del mundo empírico (Riascos, 2006; Jiménez, 2005), criterio que coincide con lo expresado por Coaguila (2017), Rojas (2014), Escudero (2013) y Zaratiegui (1999) quienes plantean que la organización es un conjunto de sistemas interactuantes conformados por procesos.

Estas organizaciones sistémicas tienen una estructura, pero ésta puede ser cambiante y adaptable en el tiempo, según sean las condiciones del entorno y de los cambios internos que se experimenten, identificándose los elementos siguientes (Narváez, Lavell y Pérez 2009):

1) Los insumos, que se reciben de los proveedores externos (extrae y asimila del entorno) son para transformarlos en un producto o resultado final.

2) Los procesos, constituidos por las interacciones sucesivas que deben existir entre las unidades que intervienen y participan dentro de la organización para transformar progresivamente y en forma conjunta los insumos, hasta obtener el producto o resultado final esperado.

3) El producto o resultado final que la organización genera responde de acuerdo a los requerimientos y las especificaciones del cliente o usuario externo.

4) El cliente o usuario del producto, es el que alimenta al sistema con sus requerimientos y las especificaciones o características del producto que se quiere lograr.

Sin embargo, habitualmente las organizaciones se asocian a empresas o movimientos sociales, pero no a un territorio o destino, sin embargo, se puede considerar que si bien las organizaciones sistémicas tienen un objetivo claro con características específicas, el destino turístico puede ser considerado una organización sistémica si se tiene en cuenta que, de igual manera, responde a estas características que las singularizan, a los elementos que la conforman y su análisis exige de un enfoque sistémico, respondiendo a objetivos definidos.

El destino turístico, es considerado un fenómeno complejo cuya definición presenta desafíos, y los autores lo enmarcan desde diferentes perspectivas de análisis, pero siempre tienen implícito la relación que se establecen entre sus componentes:

- Espacio físico o geográfico (Becker, 2014; Anjos \& Oliveira 2013; Anton et al.,2012; Vera et al., 1997 ;Bull, 1994 y Acerenza,1987)

- Producto turístico (Pearce, 2014; Marín, 2012; López \& López, 2007; Hudson, 2005; OMT, 2004; Bigné et al., 2000;Buhalis, 2000 ;Cooper, 1993)

- Visión mixta (Echarri, 2006; Barrado, 2004; OMT, 2004; Framke, 2002). 
Considerando esta diversidad de criterios se hace necesaria una mirada integrada del concepto de destino turístico que permita una lectura dinámica sobre las realidades que lo caracterizan.

El destino turístico comparte con las organizaciones sistémicas los componentes de su estructura, pero los redefine considerando los insumos como los recursos del entorno/territorio, los procesos como las interacciones de las unidades de transformación del producto, que el mismo se traduce en la oferta y los clientes devienen en los grupos de interés que son aquellos que alimentan el sistema según sus requerimientos y cuya satisfacción constituyen las salidas. En su integración deben orientarse hacia un mismo fin en el marco de una misión compartida.

Tomando estas consideraciones se define que el destino turístico es una organización sistémica, que se enmarca en un territorio, tiene límites geográficos definidos, y está conformado por partes interrelacionadas que constituyen un todo coherente, que presenta diferentes características naturales y antrópicas, formado por elementos espaciales (recursos territoriales e infraestructuras), administrativos (legislación, políticas), productivos (factores y recursos de producción, agentes, e inversión) y sociales (visitantes, turistas y comunidad), con diversidad de intereses, y que funcionan como un sistema. Condición esta que le confiere la capacidad para responder y adaptarse a los impactos del medio, aprender de sus propias experiencias y errores, poder desarrollarse mediante mecanismos propios de autorregulación y control, a partir de las dinámicas generadas por las interrelaciones que existen entre las unidades que lo integran así como sus interdependencias recíprocas en función de objetivos comunes.

De ahí que resulta viable la adaptación de los basamentos del enfoque a procesos para evaluar la sostenibilidad de los destinos turísticos, teniendo en cuenta que las interconexiones e interrelaciones que concurren en el sistema turístico se materializan en este, que, como contenedor y receptor de la actividad turística es el espacio donde se concretan las actuaciones de los componentes del sistema turístico de modo que hay una relación dual: el sistema incluye al destino y en el destino se materializa o visualiza el sistema, de modo que el destino es una expresión local del sistema turístico pudiéndose entender como tal.

El enfoque a procesos permite establecer una base objetiva para el desarrollo de investigaciones con respecto a este tema, ya que éste reconoce la modelización de los sistemas como un conjunto de procesos interrelacionados mediante vínculos de causaefecto que sustenta la visión sistémica de un destino turístico, facilitando integrar en un mismo instrumento (el mapa de proceso), el análisis de un destino turístico con sus diferentes etapas (planificación, implementación, verificación y control).

En resumen las ventajas de la inserción del enfoque a procesos en los destinos turísticos, puede consignarse en:

- Permite integrar múltiples actores de diversas naturalezas que configuran la cadena de valor del producto turístico en el destino. 
- Es una herramienta para la gestión que facilita identificar áreas de actuación en un entorno de mejora continua, permitiendo la retroalimentación, contribuyendo a la planificación y a la toma de decisiones.

- Identificación y participación de los grupos de interés, logrando un enfoque integrado hacia sus necesidades expectativas, que facilita homogeneizar el diseño de objetivos comunes en la gestión integral del destino.

- Simplificación de estructuras, enfocando el trabajo hacía una única cultura organizacional y de gestión en el destino.

- Inclusión de actividades de valor añadido que incrementan la satisfacción de las expectativas hacia los grupos de interés previamente identificados.

- Reducción de costos e incremento de la eficacia y eficiencia a partir de la mejora. (indicadores) evitando las grandes diferencias de capacidad y de asunción de riesgos financieros

Estas ventajas, conducen a la superación de las problemáticas que manifiesta desde mediados de los 90 del siglo XX el análisis tradicional de los destinos turísticos, que según diversos autores, se presentan en los ámbitos del establecimiento de objetivos, cultura organizacional, riesgos financieros y el propio desarrollo turístico (Antón et al., 2012; Zapata, 2008; Vera et al., 2011,1997; Socher, 1993).

Tabla 1. Diferencias entre el análisis tradicional de destinos turísticos y con enfoque a procesos.

\begin{tabular}{|c|c|c|}
\hline & $\begin{array}{l}\text { Gestión de destinos } \\
\text { turísticos(Tradicional) }\end{array}$ & $\begin{array}{l}\text { Gestión de destinos turisticos. } \\
\text { (Enfoquea procesos) }\end{array}$ \\
\hline $\begin{array}{l}\text { Definición de } \\
\text { Objetivos }\end{array}$ & $\begin{array}{l}\text { Diversos objetivos (atendiendo } \\
\text { a las particularidades de la } \\
\text { organización). }\end{array}$ & $\begin{array}{l}\text { Único fin común en función de las } \\
\text { necesidades y expectativas de los } \\
\text { grupos de interés logrando un } \\
\text { enfoque integrado. }\end{array}$ \\
\hline Estructuras & $\begin{array}{l}\text { Diferentes culturas } \\
\text { organizacionales y de gestión. }\end{array}$ & $\begin{array}{l}\text { Única cultura organizacional y de } \\
\text { gestión. }\end{array}$ \\
\hline $\begin{array}{l}\text { Visión de riesgos } \\
\text { financiero }\end{array}$ & $\begin{array}{l}\text { Diferencias de capacidad y de } \\
\text { asunción de riesgos financiero. }\end{array}$ & $\begin{array}{l}\text { Reducción de costos financieros e } \\
\text { incremento de la eficacia. }\end{array}$ \\
\hline
\end{tabular}

Fuente: Elaboración propia.

\section{Elementos básicos para adaptar el enfoque a procesos en la evaluación de la sostenibilidad de los destinos turísticos.}

1. Disponer de un mapa de procesos del destino, que represente los procesos, y subprocesos del mismo permitiendo establecer las interrelaciones e interconexiones que en el se producen, teniendo en cuenta la clasificación que responde a los procesos de planificación, realización del producto y servicio, gestión de recursos, y medición - análisis; como se muestra en la figura 1.

2. Reconocer el grupo de interés del destino turístico. 


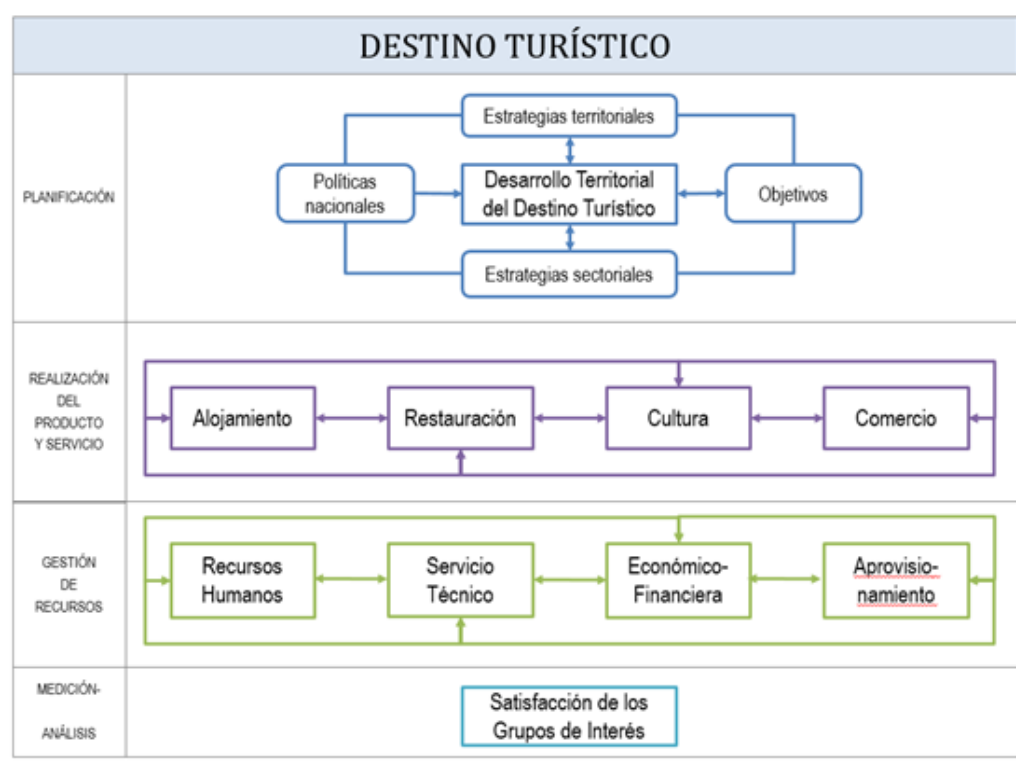

Leyenda: Conectores entre los subprocesos

Subprocesos

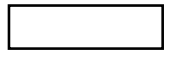

Entradas y salidas de los subprocesos

Figura 1. Mapa de proceso de un destino turístico.

Fuente: Elaboración propia.

3. Contar con un modelo de ficha para destinos turísticos por cada dimensión de la sostenibilidad: económica, ambiental y sociocultural como se muestra en la figura 2, donde se agrupan de manera sintetizada los elementos fundamentales que identifican el proceso o subproceso, teniendo en cuenta que a diferencia de las fichas de procesos que exponen criterios de medida e indicadores para evaluar la eficacia y eficiencia del proceso, en este caso se consideran criterios e indicadores de sostenibilidad y se introducen nuevos elementos con el propósito de integrar a la evaluación del proceso: un índice de control de la sostenibilidad turística por dimensiones y uno general que permita interpretar de forma global e integrada el fenómeno, es decir, valorar conjuntamente sus características multidimensionales a partir de los procesos que se producen en un destino turístico. 


\begin{tabular}{|l|l|l|}
\hline \multicolumn{3}{|c|}{ GENERALES DEL PROCESO } \\
\hline Nombre del proceso & $\begin{array}{c}\text { Fecha de } \\
\text { edición }\end{array}$ & Responsable o propietario \\
\hline Objetivo & Documentos y registros \\
\hline Entradas & Salidas \\
\hline \multicolumn{2}{|c|}{ Indicadores de sostenibilidad } \\
\hline ESPECíFICAS DE LA DIMENSIÓN DE LA SOSTENIBILIDAD \\
\hline Sostenibles & \multicolumn{2}{|c|}{} \\
\hline \multicolumn{2}{|c|}{} \\
\hline índice de control de la sostenibilidad turística dimensión ambiental \\
\hline
\end{tabular}

Figura 2 Ficha de proceso de un destino turístico para evaluar sostenibilidad.

Fuente: Elaboración propia.

A continuación, se definen los nuevos elementos que forman parte de la estructura de la ficha.

Índice de control de Sostenibilidad Turística Dimensional (ICSTD): Es el valor que permite conocer el comportamiento de cada una de las dimensiones que componen la sostenibilidad. Constituye la base para evaluar de manera simplificada y desde el punto de vista sostenible un proceso o subproceso.

Se aplica un modelo de índices no ponderados según lo planteado por de la Fuente (2013) se realizará por media aritmética. Sin embargo, los procesos de agregación que se derivan tienen como limitación más significativa la compensación de impactos que supone una suma aritmética de variables, por lo que el déficit en un indicador puede ser compensado por el plus de otro. Para minimizar el error de interpretación que esto puede suponer, no se deberá perder de vista la contribución que hace cada indicador simple al valor agregado que supone al índice de control. Además se estandariza a través de una escala de $\quad+10$ -1) moviéndose entre un rango de variabilidad $(-1 \leq \mathrm{ICST} \leq 1)$ que determina los límites de control superior e inferior en que se encuentra cada dimensión, como se describe en la tabla siguiente:

Tabla 2. Atributos de la elaboración del índice de control dimensional de sostenibilidad turística. 


\begin{tabular}{l}
$\begin{array}{c}\text { Indicadores } \\
\text { simples }\end{array} \quad$ Normalización $\quad$ Fórmula Aritmética \\
\hline
\end{tabular}

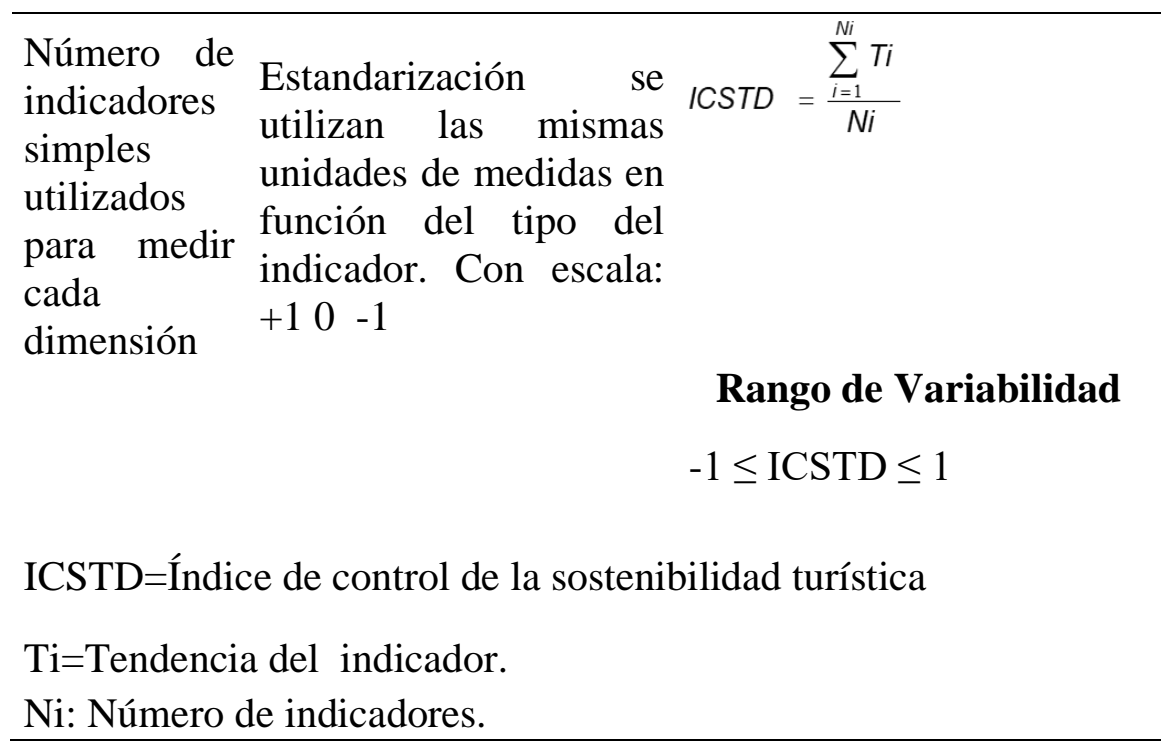

Fuente: Elaboración propia.

A partir del índice control se construye el Índice General de Sostenibilidad Turística (IGST): este permite disponer de un dato en síntesis, en forma de valor único de la sostenibilidad turística general del proceso o subproceso en cuestión. Su aplicación periódica permite reconocer la dinámica evolutiva de la sostenibilidad, la comparación entre situaciones temporales del destino, facilitando la retroalimentación, realizar propuestas de mejora y ayuda en la toma de decisiones. Los elementos del índice están representados en la tabla siguiente.

Tabla 3. Atributos del Índice general de sostenibilidad turística.

\section{Rango de evaluación Fórmula Aritmética}

$-1 \leq \mathrm{IGS} \leq 1 \mathrm{GS}=\frac{\sum_{i=1}^{N d} I C S T D}{N d}$

IGS=índice general de sostenibilidad

ICSTD = valor del índice de control de la sostenibilidad turística (por dimensión) =ICSa+ICSe+ICSsc....+ICSn

$\mathrm{Nd}=$ Total de dimensiones a evaluar

Fuente: Elaboración Propia.

Los indicadores simples se normalizan para agregarse en un Índice de control de sostenibilidad turística por dimensiones, que a su vez se combinan en subíndices para 
generar un único valor general adimensional, de modo tal que las dimensiones que se analizan se consideran igualmente prioritarias y están equilibradas en sus componentes por media aritmética de los índices normalizados de cada una de las tres dimensiones.

El proceso o subproceso en cuestión se evalúa a través del análisis estadísticomatemático, utilizando el software EXCEL para calcular las tendencias de los indicadores, el índice de control por dimensiones y un índice general de la sostenibilidad turística. Para lo cual se define que la tendencia del indicador tendrá una escala de (-10 1), donde 1 representa la tendencia positiva, 0 estancado y -1 negativo; que determinarán el comportamiento del criterio de sostenibilidad, para en un segundo momento calcular el índice de control por dimensiones y el índice general de sostenibilidad turística.

\section{Conclusiones}

- Las características, premisas y funcionamiento que distinguen a las organizaciones, pueden ser reconocidas en un territorio o destino turístico, por lo que este puede ser considerado una organización sistémica, en el cual pueden aplicarse los principios que rigen los basamentos del enfoque a procesos.

- La trayectoria metodológica de la investigación permitió reconocer que para la adaptación de los basamentos del enfoque a procesos en la evaluación de la sostenibilidad de un destino turístico, se hace necesario disponer del mapa de proceso del destino, reconocer su grupo de interés y elaborar una estructura de ficha de proceso por cada dimensión a la que se le introducen nuevos elementos: el índice de control de la sostenibilidad turística por dimensiones y uno general que permite interpretar de forma global e integrada el fenómeno.

\section{Referencias bibliográficas}

Acevedo, J.; M., Gómez, A.,Urquiaga, (2011) Modelo de valor de los procesos, base para la gestión económica - financiera integrada" Revista Nueva Empresa , 7, 2, 23-30, pp.1682-2455.

Acevedo, J., 1995, Análisis, mejoramiento y control de la gestión de los procesos, Universidad Tecnológica (CUJAE), La Habana. Anton, S et al., (2012).El reto de reinventar los destinos, pp. 17-150.

Anton, S y F. González (Coordinadores) (2007) A Propósito del Turismo. La construcción social del Espacio Turístico, Editorial UOC, Barcelona, 346 pp.

Anton, S y F. González (Coordinadores) (2005) Planificación Territorial del Turismo, Editorial UOC, Barcelona, 216 pp.

Barrado, D. (2015) Recursos turísticos y atractivos turísticos: conceptualización, clasificación y valoración, Cuadernos de Turismo, nº 35, pp. 335-357.

Barrado, D. A., 2004, El concepto de destino turístico: una aproximación geográfico territorial: Estudios Turísticos p. 160. 
Bigné, E. et al (2001). "Marketing de Destinos Turísticos”. ESIC Editorial, Madrid. España.

Cosa, M (2011) Gestión y estructura organizativa de las destination management organizations: un enfoque económico-empresarial Universidad de Bari, Italia,19 pp.

Cosa, M. (2009): L'impresa post-capitalistica e il suo ruolo nell'economia moderna. Approccio sistemico e teoria organicistica a confronto, Rirea, Roma.

Coaguila,A. (2017)Propuesta de implementación de un modelo de gestión por procesos y calidad en la empresa o\&c metals s.a.c. Tesis en Opción al Título de Ingeniero Industrial. Facultad de Ingeniería y Computación, universidad católica, San Pablo.

Coelho, P, Mascarenhas, A., Vaz, P., Dores, A., TB, Ramos (2010). A framework for regional sustainability assessment: developing indicators for a Portuguese region. Sustainable Development. Da Silva ,M.,C. Pasa (2013) Aplicação do Tourism Ecological Footprint Method para avaliação dos Impactos Ambientais do Turismo em Ilhas: um estudo em Fernando de Noronha, Revista Brasileira de Pesquisa em Turismo. São Paulo, 7(2), pp. 220-238.

Delgado, A. (2013) Impacto del Cambio Climático en la Demanda Turística. Tesis en Opción al Título de Doctor en Ciencias Geográficas, Universidad de La Habana, Cuba.

Dredge D. (1999) Planificación y diseño de Destinos Turísticos, Annals of Tourism Research en español, Vol. 1 N², pp. 394 - 414

Echarri, M. (2006): Análisis Geográfico del Turismo en Ciudades Patrimoniales Cubanas. Tesis en Opción al Título de Doctor en Ciencias Geográficas, Universidad de La Habana, Cuba.

Escudero, D. (2013): “Flujograma de procesos", VAAC, Gerencia de Administración y Finanzas, 12/8/2012, 18 pp.http://www.slideshare.net.

Fuente, S. de la (2013) Números e Índices, Facultad de Ciencias Económicas y Empresariales, UAM, España

Jiménez, A. (2005) Una aproximación a la conceptualización del turismo desde la teoría general de sistemas. México, 90 pp.

Narváez, L., Allan Lavell, Gustavo Pérez Ortega, 2009, La gestión del riesgo de desastres: Un enfoque basado en procesos: Lima, Perú. 
NC ISO 14001:2015 "Sistemas de Gestión Ambiental. Requisitos con orientación para su uso". Oficina Nacional de Normalización. Cuba.

Organización de las Naciones Unidas(1987) Nuestro Futuro Común o Informe Brutland.. Disponible en: http://www.tij.uia.mx/elbordo/vol05/. (consulta: octubre del 2017).

Organización Mundial del Turismo (OMT) (2004). "Gestión de la saturación turística en sitios de interés natural y cultural. Guía práctica”, España.

Pearce, D. (2016) Modelos de gestión de destinos. Síntesis y Evaluación, Estudios y Perspectivas en Turismo Volumen 2, pp. $1-16$.

Riascos , J.(2006)De la estructura por funciones al enfoque basado en procesos y a la visión sistémica de la Organización. Revista Ciencias Estratégicas, vol. 14, núm. 15, enero-junio, Universidad Pontificia Bolivariana Medellín, Colombia, pp. 33.42

Rojas, D. (2014) Modelo para la implementación de un sistema de gestión integral alineado a la estrategia empresarial de la organización.Ensayo presentado para el Diplomado en Sistemas de Gestión de Calidad. Universidad Militar, Nueva Granada.

Rummler, G. y Brache, A, (1995), Improving Performance. How to Manage the White Space on the Organization Chart, Jossey - Bass Publishers, Second Edition. United States, San francisco, California.

Salinas, E. et al., (2008). Metodologías para la evolución de la sustentabilidad teritorial: el uso de indicadores en destinos turísticos de Cuba. Boletín de la R. S. G., CXLIV, págs. $70-100$.

Sharpley, R. (2010). Tourism and Sustainable Development: Exploring the Theoretical Divide. Journal of Sustainable, 8(1), 1-19.

Tarlombani da Silveira, M. A. “Turismo Y Sustentabilidad: Entre el discurso y la acción”. Universidad Federal de Paraná Curitiba. Brasil. 2005.

Thompson, I. (2012). Definición de organización desde distintos puntos de vista». http://www.elergonomista.com/dom02.html, [14/07/2017].

Sirakaya, E., Jamal, T., \& Choi, H. S. (2001). Developing indicators for destination sustainability. In D. B. Weaver (Ed.), The encyclopedia of ecotourism (pp. 411432). New York: CAB International.

Ulacia, Z. (2007) La gestión de procesos en la hospitalidad. Editorial Centro de Estudios Turísticos. Ciudad de La Habana. Cuba. 
Torres, H. L., (2016), Propuesta de esquema metodológico para la evaluación de la sostenibilidad del desarrollo turístico de destinos caso La Habana, Universidad de La Habana, La Habana.

Vera, J. F. (Coord.), López Palomeque, F., Marchena, M., \& Anton, S. (2013). Análisis territorial del turismo y planificación de destinos turísticos. Valencia: Tirant Humanidades.

Zaratiegui, J. R., 1999, La gestión por procesos: su papel e importancia en la empresa: Economía Industrial, v. VI: España. 


\section{PARA CITAR EL ARTÍCULO INDEXADO.}

Robert Beatón, M. O., Echarri Chávez, M., \& Acevedo Suárez, J. A. (2021). La evaluación de la sostenibilidad de los destinos turísticos en su relación con el enfoque a $\begin{array}{llll}\text { procesos. } & \text { Explorador } & \text { Digital, } & 5(1),\end{array}$ https://doi.org/10.33262/exploradordigital.v5i1.1506

\section{\Cigncia}

El artículo que se publica es de exclusiva responsabilidad de los autores y no necesariamente reflejan el pensamiento de la Revista Explorador Digital.

El artículo queda en propiedad de la revista y, por tanto, su publicación parcial y/o total en otro medio tiene que ser autorizado por el director de la Revista Explorador Digital.
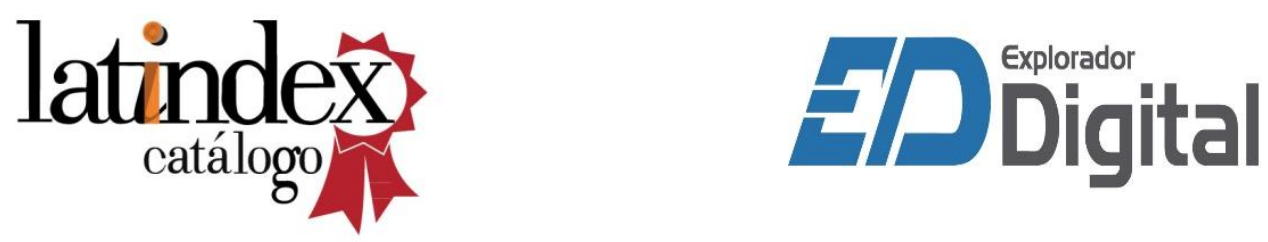